\title{
Remote auroral activity detection and modeling using low frequency transmitter signal reception at a midlatitude site
}

\author{
E. D. Schmitter \\ University of Applied Sciences Osnabrueck, 49076 Osnabrueck, Germany \\ Received: 10 August 2010 - Accepted: 27 September 2010 - Published: 30 September 2010
}

\begin{abstract}
The low frequency propagation conditions along the path from Iceland to Germany $\left(52^{\circ} \mathrm{N} 8^{\circ} \mathrm{E}\right)$ using the NRK/TFK $37.5 \mathrm{kHz}$ transmitter $\left(63.9^{\circ} \mathrm{N} 22.5^{\circ} \mathrm{W}\right)$ prove as an easy to monitor and reliable proxy for north auroral activity. Signal processing using wavelet decomposition allows for quantitative activity level estimations. Calibration is based upon NOAA POES auroral activity data. Using an auroral oval model for the local intensity distribution of solar energetic particle precipitation and a wave propagation model ionospheric D-layer height decreases along the path can be derived. This in turn gives a hint to the low latitude extension and intensity of the auroral electrojet currents that can be responsible for communication and power systems failures.
\end{abstract}

Keywords. Ionosphere (Auroral ionosphere; Modeling and forecasting; Particle precipitation)

\section{Introduction}

Remote detection of auroral activity using moderately elaborate equipment is a good complement to in situ measurements and satellite based recordings, especially with regard to the lower ionosphere behaviour which cannot be accessed "from top" and also with regard to nearly $24 / 7$ availability of data covering the same propagation range. We follow and extend previous work done by Cummer et al. (1996), Cummer et al. (1997), Kikuchi and Ohtani (1986), Peter et al. (2005). Their work indicated that VLF phase and amplitude anomalies and effective D-layer reflection height decrease is well correlated with an increase of high energetic $(E \geq 300 \mathrm{keV})$ electron precipitation during expansion of the equatorward boundary of the auroral oval crossing VLF

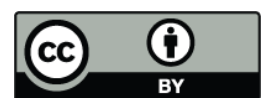

Correspondence to: E. D. Schmitter (e.d.schmitter@fh-osnabrueck.de) propagation paths. Location and intensity of the auroral electrojet which is coupled to this boundary are not only of geophysical interest but important for the assessment of possible communication and electric power systems failure risks. The organization of the paper is as follows: in Sect. 2 we describe the characteristic variations of the signal amplitude of the NRK/TFK $37.5 \mathrm{kHz}$ transmitter $\left(63.9^{\circ} \mathrm{N} 22.5^{\circ} \mathrm{W}, L=5\right.$, Iceland) at the receiver site $\left(52^{\circ} \mathrm{N}, 8^{\circ} \mathrm{E}, L=2.2\right.$, NW Germany) and its wavelet based analysis used for the estimation of the auroral activity level. Section 3 deals with the auroral oval model and the propagation model needed for the calculation of the effective ionospheric reflection height decreases caused by precipitations related to auroral oval expansion. The last chapter presents results and conclusions.

\section{Estimating auroral activity}

For getting information about auroral activity we monitor the signal amplitude of (very) low frequency MSK (Minimum Shift Keying) transmitters within the subpolar auroral zone. The Tx station with call sign JXN (Norway, $67^{\circ} \mathrm{N} 14^{\circ} \mathrm{E}$ ) transmits only sporadically, whereas the Tx station with call sign NRK/TFK at Grindavik, Iceland $\left(63.9^{\circ} \mathrm{N} 22.5^{\circ} \mathrm{W}\right.$, $L=5$ ) usually transmits $24 / 7$ with few dropouts. Additionally near to this site the Leirvogur magnetometer is situated so that we can assess the geomagnetic activity at the transmitter site (URL: www.raunvis.hi.is/ halo/lrv.html) caused by variations of the location and the intensity of the auroral electrojet current system - often coincident with the low latitude boundary of high energy auroral particle precipitation Cummer et al. (1997). We use 2 receivers at an average mid latitude location of $\left(52^{\circ} \mathrm{N}, 8^{\circ} \mathrm{E}, L=2.2\right)$ with a distance of $30 \mathrm{~km}$ and a great circle distance to the transmitter of $2210 \mathrm{~km}$. Comparing the signals of the two receivers we can discard local disturbances. The receivers use coils with a ferrite core as sensors for the horizontal magnetic field 


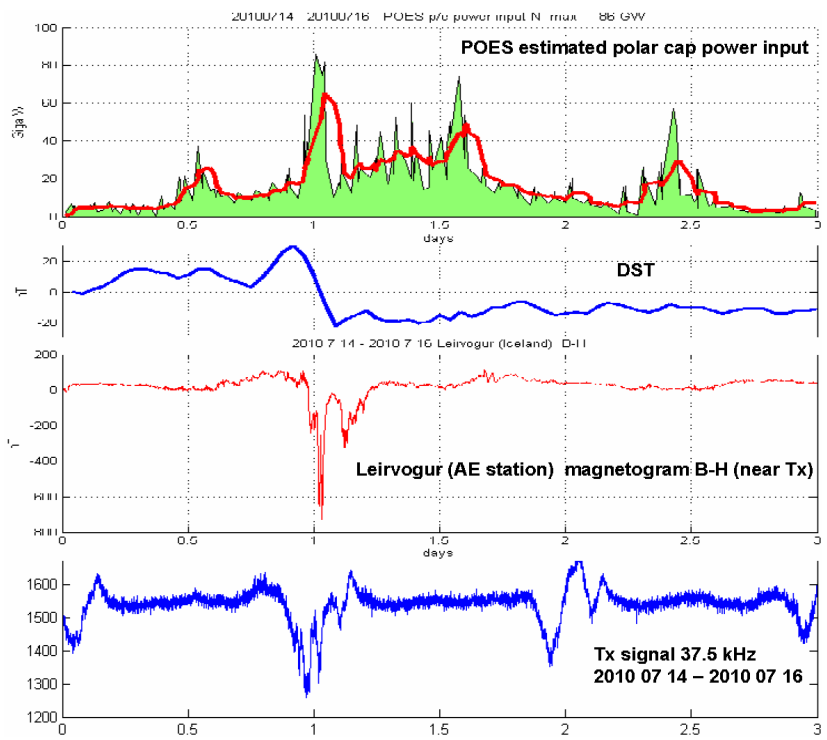

Fig. 1. Top down: NOAA POES auroral activity data, DST index, horizontal component Leirvogur magnetogram (sensitive to location and intensity of the auroral electrojet current system) and Tx signal amplitude monitored at 52N 8E (sensitive to high energy particle precipitation along the propagation path).

component of the signal. The broadband preamplified signal is fed via a soundcard into a computer, where the Tx signal is extracted via Fast Fourier Transform (FFT) and further analysed. Figure 1 shows an example recording for the 3 days 14 July 2010 to 16 July 2010 (lowest of the 4 traces). During this time some minor auroral activity took place nevertheless showing up in a disturbed night time signal clearly to be seen between the first and the second day. Variations of location and intensity of the auroral electrojet current system clearly show up in the Leirvogur horizontal magnetogram (3. trace). The first trace shows the total polar cap power input in GigaWatt as estimated by the NOAA POES satellites (NOAA 15-19, Polar Orbiting Environmental Satellites, URL: www.swpc.noaa.gov/pmap). Auroral activity also is a proxy for global geomagnetic activity, represented here by the Kyoto DST index (2. trace) where the drop event coincides with the start of the main auroral activity.

To quantify the information content of the signal recordings for the discrimination of quiet and disturbed parts of the time series a wavelet decomposition is done. We decided for the Morlet wavelet decomposition (Lewalle, 1995). Compared to the many wavelet transforms in use this one is especially suited to detect pseudo-periodicities. The Morlet is a wave packet and in frequency space Morlet transform acts like a bunch of bandpass filters moved along the time series to be analyzed. Figure 2 shows the signal received on 2 May 2010 and below the amplitude of pseudo periods from (nearly) 0 (bottom) to $1 \mathrm{~h}$ (top). Besides the terminator struc-

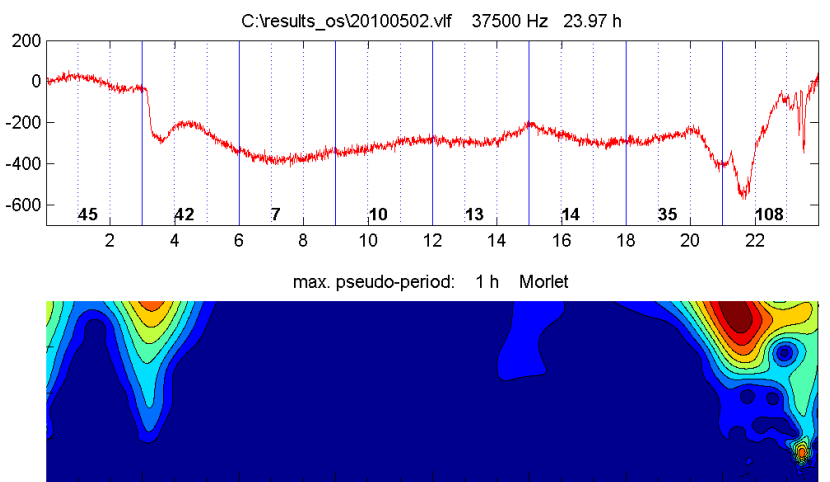

Fig. 2. Monitored signal amplitude and wavelet decomposition (bottom of wavelet decomposition: period 0 , top: period $1 \mathrm{~h}$ ).

tures we see the signature of a disturbance after 23:00 UT with characteristic pseudo periods between 15 and $30 \mathrm{~min}$.

As a measure for the total pseudo periodic activity $A(\mathrm{UT})$ of the signal within a $3 \mathrm{~h}$ time slot starting at time UT we use the sum of all wavelet coefficients (absolute values) within that slot:

$A(\mathrm{UT})=\sum_{3 \mathrm{~h} \text { pseudo periods }<1 \mathrm{~h}} \mid$ Morlets $\mid$

To derive an index for the auroral activity, the pseudo periodic activity of a quiet day directly neighbouring the active days is subtracted. Otherwise the wavelet signatures of the sunrise/set terminators would distort the result.

We model the polar activity level $a_{c}$ estimated from our low frequency signal amplitude recordings by

$a_{c}(\mathrm{UT})=\alpha\left(A(\mathrm{UT})-A_{\text {quiet }}(\mathrm{UT})\right)(1+2 \cos (z))$

$z$ is the suns zenith angle halfways at the propagation path. By the factor $(1+2 \cos (z))$ we empirically take into account that day over the signal is less sensitive to additional ionization effects than during night. The scale factor $\alpha$ depends on the details of the Morlet transform and is calibrated by comparing results to the NOAA POES auroral activity data.

Now further analysis has the goal to extract additionally to the estimated auroral activity levels the effective lower ionospheric height variations along the Tx-Rx path from the data. For this we need a model of the auroral oval particle precipitation intensity in dependence on latitude and longitude and additionally a wave propagation model.

\section{Modeling ionospheric height variations}

Based on the estimated polar activity level we can calculate the ionizing effect along a given propagation path through the polar and subpolar domain using an auroral oval model. We describe it in the following subchapter. Afterwards the wave propagation model is explained. 


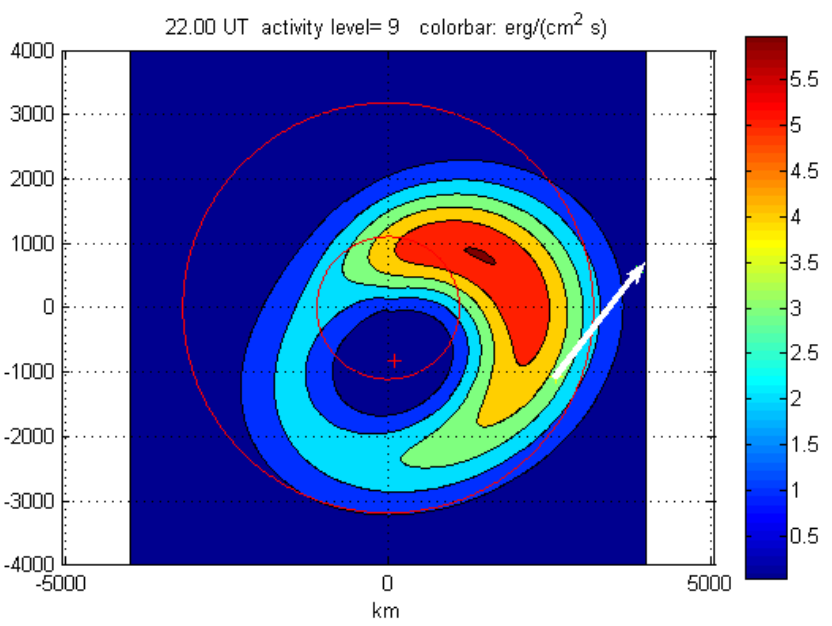

Fig. 3. Auroral oval model for activity level 9, position at 22:00 UT. Indicated are the 60 and 80 degree latitude circles, the position of the geomagnetic pole and the propagation path from $\left(63.9^{\circ} \mathrm{N}\right.$, $\left.22.5^{\circ} \mathrm{W}\right)$ to $\left(52^{\circ} \mathrm{N} 8^{\circ} \mathrm{E}\right)$ as white arrow.

\subsection{The auroral oval model}

For an activity level $a_{c}$ at universal time UT the particle precipitation intensity is modelled by an elliptic Gaussian according to

$I$ (lat, long, UT, $\left.a_{c}\right)=I_{0} w^{2} e^{-\frac{w}{2 \sigma_{a}^{2}}}$

With the projected coordinates $x=r \cos ($ long), $\quad y=$ $r \sin$ (long), $r=r_{e} \cos$ (lat), $r_{e}=6371 \mathrm{~km}$ as components of a vector $\boldsymbol{r}^{T}=(x, y)$ we define the vector $\boldsymbol{r}_{n}=R_{\mathrm{UT}}(\boldsymbol{r}-$ $\boldsymbol{r}_{\text {mag pole }}$ ) which centers the coordinates at the magnetic pole and rotates according to the universal time $U T$ using the usual 2-dimensional rotation matrix with UT expressed as rotation angle. $w=\boldsymbol{r}_{\boldsymbol{n}}{ }^{T} Q \boldsymbol{r}_{\boldsymbol{n}}$ is the bilinear form of $\boldsymbol{r}_{n}$ scaling these coordinates to an elliptic figure with semiaxes $a=\left(1590+130 a_{c}\right) \mathrm{km}$ and $b=\left(1270+100 a_{c}\right) \mathrm{km}$ with the scaling matrix $Q=\left(1 / a^{2} \quad 0 ; 01 / b^{2}\right)$ (Chen et al., 2008). Chen et al. (2008) use the natural log of the AE-index instead of the auroral activity index $a_{c}$ in the linear combinations for the semiaxes $a$ and $b$.

$I_{0}$ and $\sigma_{a}$ are calibrated as functions of the activity such that the oval shapes according to the POES auroral activity maps (www.swpc.noaa.gov/pmap/) are reproduced and to satisfy:

$\int_{\text {polar-cap }} I($ lat, long $) d \Omega=I_{\text {total }}$

where $I_{\text {total }}$ is the total estimated power input into the north polar cap area provided by the NOAA POES data. $I_{\text {total }}$ in GigaWatt is related to the activity level $a_{c}$ according to

$I_{\text {total }}=e^{a_{c} / 2}$

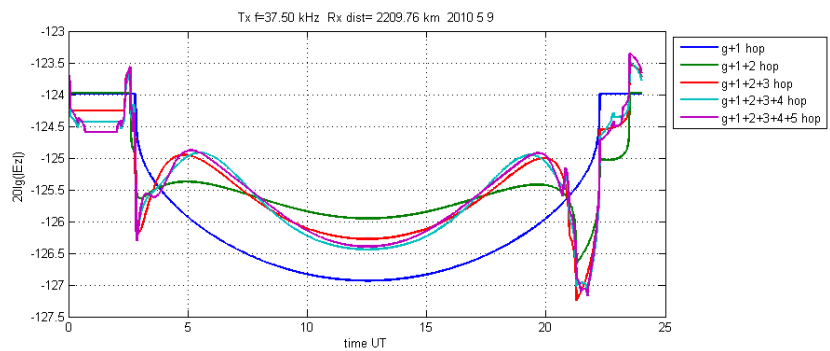

Fig. 4. Model calculations for ground wave + multi hop propagation. Signal amplitude calculations of the $37.5 \mathrm{kHz} \mathrm{Tx}$ at the receiver site $\left(52^{\circ} \mathrm{N}, 8^{\circ} \mathrm{E}\right.$, great circle distance $\left.d=2210 \mathrm{~km}\right)$ converges sufficiently with $n=5$ hops.
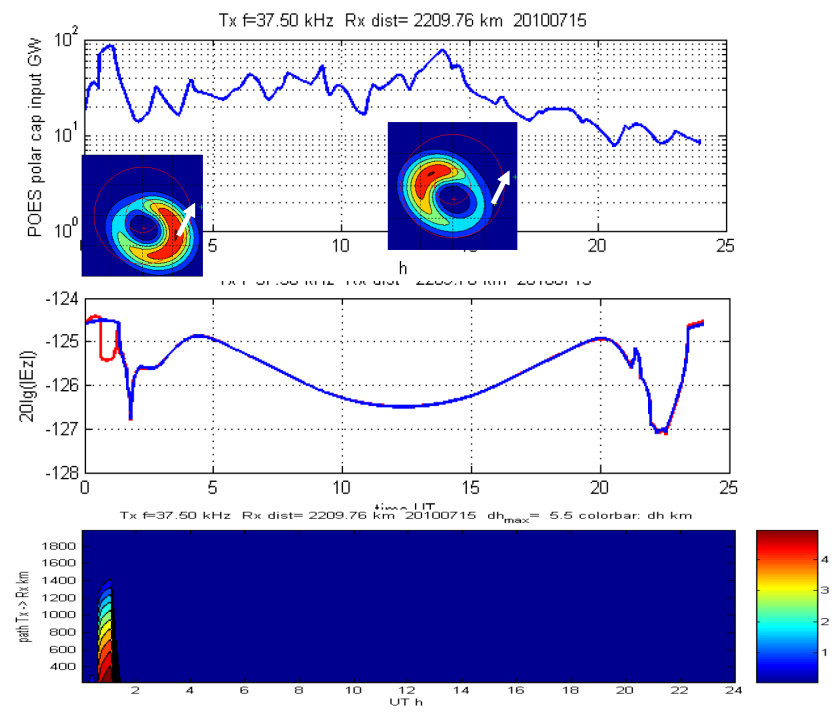

Fig. 5. POES polar cap power input for 15 July 2010, model calculation (blue: undisturbed, red: disturbed) and derived reflection height variation along propagation path. Polar impact intensity is high at around 01:00 UT and again around 14:00 UT, however only around the first time the auroral oval intensity distribution is high enough along the propagation path (white arrow) to produce significant height reduction - in this case with a maximum of $5.5 \mathrm{~km}$ at the transmitter site (bottom line) decreasing slowly along the propagation path.

The width parameter $\sigma_{a}$ depends on the activity level $a_{c}$ and on the time UT letting the small width and low intensity part of the oval point to the sun.

Figure 3 shows as an example the auroral oval intensity distribution for activity level $a_{c}=9$ at 22:00 UT. Indicated are the 60 and 80 degree latitude circles, the position of the geomagnetic pole and the relevant propagation path from $\left(63.9^{\circ} \mathrm{N}, 22.5^{\circ} \mathrm{W}\right)$ to $\left(52^{\circ} \mathrm{N} 8^{\circ} \mathrm{E}\right)$ as white arrow. 


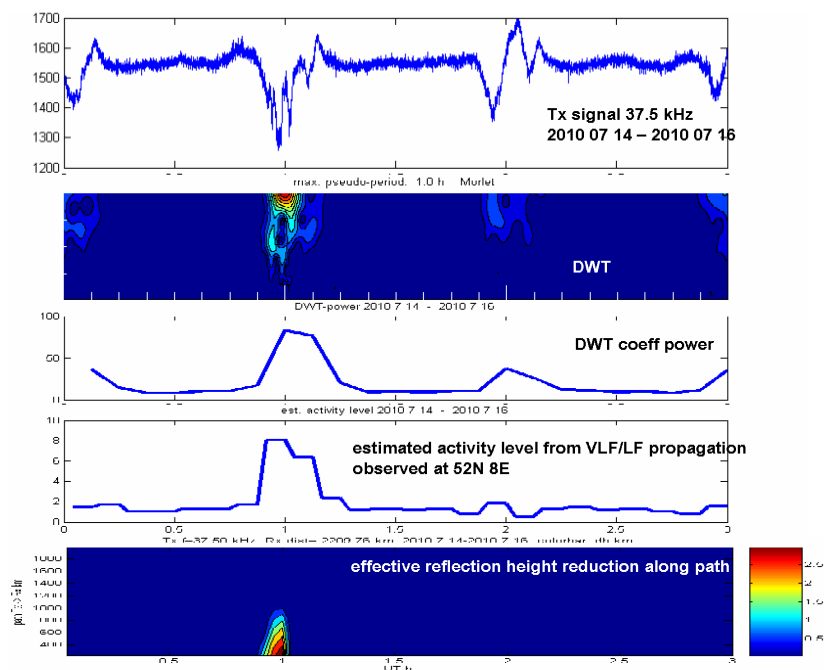

Fig. 6. For the 3 days 14 July 2010-16 July 2010: from top to bottom: Observed signal amplitude, wavelet (Morlet) decomposition with pseudo periods from nearly 0 to $1 \mathrm{~h}$, sum of absolute values of the Morlet coefficients in $3 \mathrm{~h}$ slots, estimated auroral activity index, ionospheric reflection height reduction (with a maximum of $3 \mathrm{~km}$ near the transmitter site in this case) along path (bottom line of height decrease display: Tx, top line: $\mathrm{Rx}$ ).

\subsection{The wave propagation model}

Within ray theory the electric field amplitude of a vertical Hertzian transmitter dipole is described as the sum of a ground wave contribution and multiply reflected sky waves (TM-mode, ordinary ray) following Volland (1994), Wait (1998), Yoshida et al. (2008):

$$
E_{z}=A \frac{F(d, f, \sigma)}{d}+A \sum_{j=1}^{n} \frac{B_{j}}{L_{j}} e^{i k\left(L_{j}-d\right)}
$$

The coefficient $A$ depends on transmitter power and antenna radiation efficiency, $F(d, f, \sigma)$ is the ground wave attenuation coefficient, depending on great circle distance $d, \mathrm{TX}$ frequency $f$ and ground conductivity $\sigma, F=1$ for a perfectly conducting ground. $L_{j}$ is the path length of a $j$-hop wave. $k=\frac{2 \pi}{\lambda}$ is the wavenumber. The $B_{j}$ contain the ground and ionoshperic reflection coefficients. The sky wave path lengths and the ionospheric reflection coefficients depend on the effective ionospheric reflection height $h$. In the D-layer effective ionospheric reflection is reduced by electron-neutral collisions which is taken into account via an exponential lowering of the reflection coefficient with decreasing height. Figure 4 displays the signal amplitude of the $37.5 \mathrm{kHz} \mathrm{Tx}$ at the receiver site $\left(52^{\circ} \mathrm{N}, 8^{\circ} \mathrm{E}\right)$ with $d=2210 \mathrm{~km}$. The result converges sufficiently with using $n=5$ hops. The reflection height at a specific location along the propagation path varies with the sun zenith distance angle $z$. We use:

$h=h_{\text {night }}-d h_{\text {quiet }} \cos ^{m}(z)-d h_{\mathrm{SEP}}$

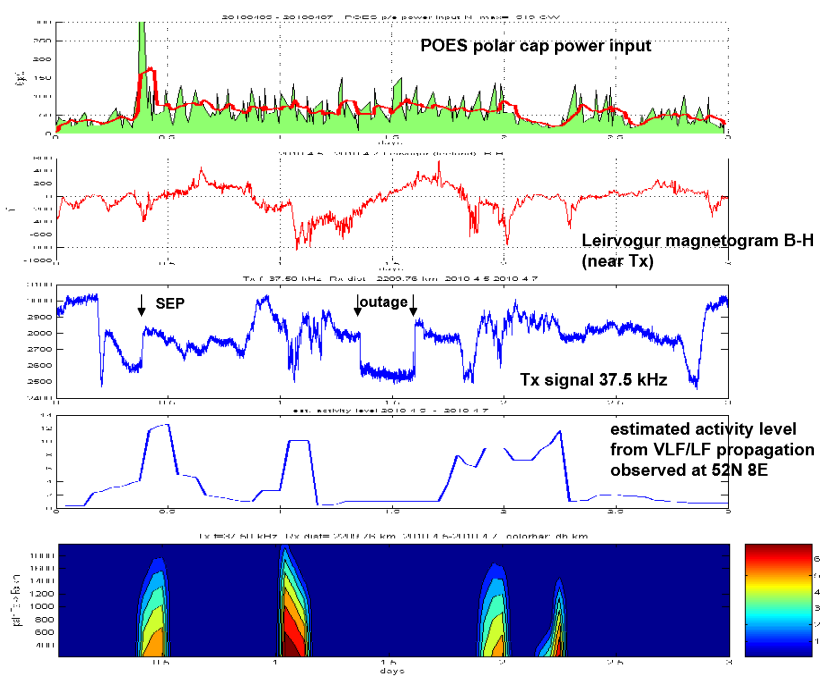

Fig. 7. For the 3 days 5 April 2010-7 April 2010: POES polar cap power input, Leirvogur magnetogram (horizontal component), observed signal, from observed signal estimated activity level, ionospheric reflection height reduction up to $7 \mathrm{~km}$ along propagation path (bottom: Tx, top: Rx). Special features: SEP onset at first day showing up in the day time observations leading to a high activity index, Tx drop out on the second day which has to be discarded for the activity level and height variation calculations.

$h_{\text {night }}, d h, m$ depend on Tx-frequency,Tx-latitude and season. For the NRK $/$ TFK Tx we used $h_{\text {night }}=86 \mathrm{~km}, d h_{\text {quiet }}=$ $14 \mathrm{~km}$ and $m=0.2$ for the summer time for a good reproduction of the observed signal amplitude in a ionospheric quiet situation. Ionization enhancement during solar energetic particle events (SEPs) is modeled by a further height reduction $d h_{\text {SEP. }}$

At given time UT and auroral activity level $a_{c}$ the height reduction at (lat, long) is modeled with

$d h_{\mathrm{SEP}}=d h_{0} \lg \left(I\left(\right.\right.$ lat, long, $\left.\left.\mathrm{UT}, a_{c}\right)\right)$

for intensities $I>1$ and $d h_{\mathrm{SEP}}=0$ otherwise.

The coefficient $d h_{0}$ is calibrated by comparing model propagation calculations to recorded data. With the auroral intensity $I$ in $\mathrm{erg} /\left(\mathrm{s} \mathrm{cm}^{2}\right)$ (to be consistent with NOAA POES data) we preliminarily found $d h_{0}=8 \mathrm{~km}$.

Figure 5 displays the POES polar cap power input for 15 July 2010 (upper trace), our model calculation (2. trace: blue: undisturbed, red: disturbed) and (3. trace) the derived reflection height variation along propagation path. We notice the influence of position and extension of the auroral oval: the impact intensity is high at around 01:00 UT and again around 14:00 UT, however only around the first time the auroral oval power input is high enough along the propagation path (respectively indicated by a white arrow) to produce significant reflection height reduction of the lower ionosphere. 

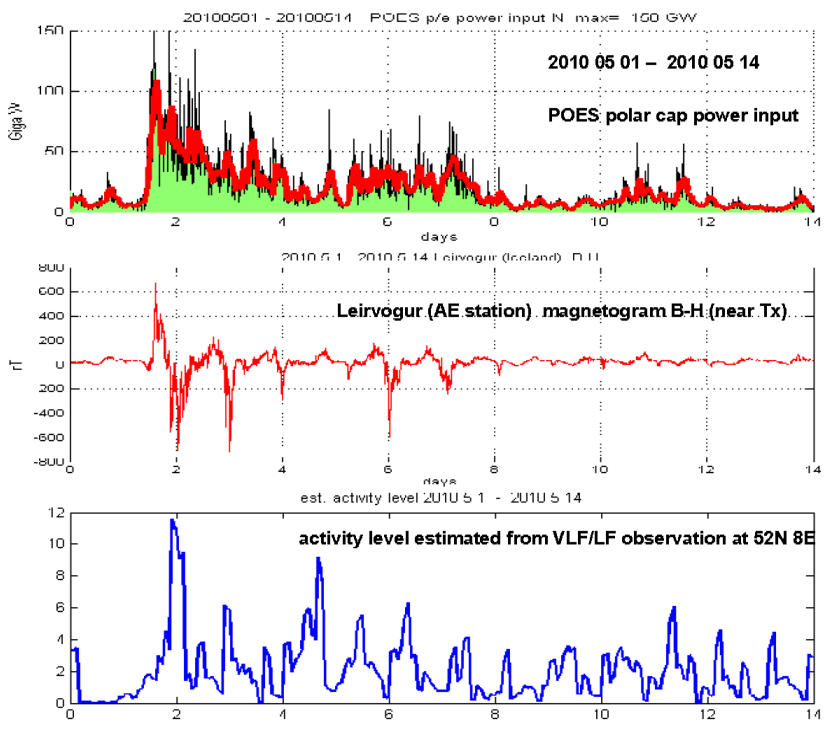

Fig. 8. 14 days period 1 May 2010-14 May 2010: POES polar cap power input, Leirvogur magnetogram (horizontal component), north polar auroral activity level estimated from observed VLF/LF signal.

\section{Results and conclusions}

Referring again to Fig. 1 as an example auroral activity caused by energetic particle precipitation massively influences propagation conditions not only of trans polar propagation paths but also paths out of the subpolar domain to lower latitudes during equatorward auroral oval expansions. The daily swing of the precipitation power input oval together with its extension and scale factor quantify the effect in time and intensity. Observations within the first year of the new solar cycle show that activity with a total polar cap input reaching and exceeding $30 \mathrm{GW}$ (corresponding to an activity level of 6.8) safely leaves its fingerprint in the night time low frequency signal. During night time two effects come together along the propagation path: the bulge of the auroral oval is at the transmitter site (the night time maxima also show up in the Leirvogur magnetograms, see Figs. 1, 8) and D-layer attenuation is strongly reduced. However strong solar energetic particle (SEP) events can be detected in the signal also during day time as is shown by Fig. 7 where a precipitation onset (shortly exceeding $500 \mathrm{GW}$ input power according to the NOAA data: 5 April 2010 09:07:39 METP02) takes place day over on the first of the 3 days (5 April 2010). The day time precipitation signature can resemble that of a SID (sudden ionospheric disturbance by X-rays of a solar flare). Whether the latter was the causing reason of course can be decided quickly by having alook at the NOAA GOES X-ray data for example.
Quantifying the disturbance effects on the signal amplitudes wavelet (Morlet) decomposition proved as a reliable tool and taking into account quiet day behavour an estimated (north) auroral activity level can be derived. This information together with an auroral oval model and a propagation path calculation is used to estimate the lower ionosphere height decrease along the propagation path (cp. Figs. 6, 7, 8) that in turn can be expressed by electron density variations. The height decrease along the propagation path also is a proxy for the mid latitude extension of the auroral electrojet current system. Adapting model calculations to recorded data shows a logarithmic dependence of reflection height decrease with local power input intensity according to Eqs. (8). Referring to a frequency of $37.5 \mathrm{kHz}$ we found $8 \mathrm{~km}$ decrease for an input of $10 \mathrm{erg} / \mathrm{cm}^{2} \mathrm{~s}=10 \mathrm{~mJ} / \mathrm{m}^{2} \mathrm{~s}$.

A refinement of the models and parameter calibrations is subject of ongoing research.

Acknowledgements. Topical Editor K. Kauristie thanks one anonymous referee for her/his help in evaluating this paper.

\section{References}

Chen, A., Li, J., Yang, G., and Wang, J.: Calculating Auroral Oval Pattern by AE Index, Acta Meteorologica Sinica, 22, 91-96, 2008.

Cummer, S. A., Bell, T. F., Inan, U. S., and Zanetti, L. J.: VLF remote sensing of the auroral electrojet, J. Geophys. Res. (USA), 5381-5389, 1996.

Cummer, S. A., Bell, T. F., Inan, U. S., and Chenette, D. L.: VLF remote sensing of high-energy auroral particle precipitation, J. Geophys. Res. (USA), 7477-7484, 1997.

Kikuchi, T. and Ohtani, A.: IV. Ionospheric Disturbances; 8. Propagation Disturbances of VLF Radio Waves on the Trans-Polar Paths, J. Radio Res. Laboratory, 33, special issue No. 1, 203217, 1986.

Lewalle, J.: Tutorial on Continuous Wavelet Analysis of Experimental Data, URL: www.ecs.syr.edu/faculty/lewalle/tutor/tutor. html, Syracuse University, 1995.

Peter, W. B., Chevalier, M., and Inan, U. S.: Subionospheric VLF measurements of the effects of geomagnetic storms on the midlatitude D-region, 11th International Ionosphere Effects Symposium, A070, 3-5 May 2005, pp. 1-8, 2005.

Volland, H.: Longwave Sferics Propagation within the Atmospheric Waveguide, Chapter 3 of Handbook of Atmospheric Electrodynamics, vol. II, edited by: Volland, H., CRC Press, 1995.

Yoshida, M., Yamauchi, T., Horie, T., and Hayakawa, M.: On the generation mechanism of terminator times in subionospheric VLF/LF propagation and its possible application to seismogenic effects, Nat. Hazards Earth Syst. Sci., 8, 129-134, doi:10.5194/nhess-8-129-2008, 2008.

Wait, J. R.: The Ancient and Modern History of EM Ground-Wave Propagation, IEEE Antennas and Propagation Magazine, 40(5), 7-24, 1998. 